\title{
ELEMENTOS FORMALES DE LOS DECRETOS DE PROXENÍA GRIEGOS
}

\author{
Mercedes Vilchez
}

Universidad de Sevilla

Lo que desarrollo aquí son algunas ideas provisionales, a modo de anticipo y muy resumidas, de un estudio más amplio que tengo en proyecto sobre los decretos de proxenía griegos desde el punto de vista formal.

Este proyecto tiene por objetivo estudiar dos aspectos:

1. El número de elementos que integran los decretos de proxenía y el orden de esos elementos según época y dialectos.

2. La estructura sintáctica de los diferentes elementos en las distintas épocas y dialectos.

Estos documentos han sido objeto de estudio desde el punto de vista histórico de la institución. Hay una reseña con comentarios muy buenos de estos estudios con el título «Rassegna di studi sulle prossenie greche» publicada en RFIC XCVII, 1969, pp. 494-501 hecha por Virgilio Biabio. Pero no se han estudiado - sincrónica y diacrónicamente- los aspectos de lengua y estilo.

Ahora voy a tratar solamente del número de los elementos que integran los decretos de proxenía y de su orden, según épocas y dialectos.

Los puntos de vista que voy a desarrollar aquí se basan en el estudio realizado sobre un muestreo hecho a partir de 100 textos seleccionados de todos los dialectos y de fechas comprendidas entre el siglo V y el siglo II a. J.C. ${ }^{1}$, y además sobre el Catálogo de proxenías de Delfos que recoge la edición de Dittemberger ${ }^{2}$.

El número total de elementos de que pueden constar es de 10. A cada elemento les aplico una signatura, que es arbitraria, en número o letra. Este número de elementos es el que se enumera a continuación:

1 Hemos extraído el material de las partes correspondientes de Inscriptiones Graecae (IG), Berlín y de Schwyzer E., Dialectorum Graecarum Exempla Epigraphica Potiora, Leipzig, 1960.

2 Dittemberger Sylloge Inscriptiones Graecarum, Hildesheim, 1960, vl. I. 


\section{Invocación.}

A. Causa que promueve la concesión de la proxenía.

B. Persona jurídica.

C. Texto de la ley.

D. Ejecución.

E. Fecha.

F. Finalidad.

G. Garantes.

2. Objeto jurídico.

3. Proponente.

De lo que he estudiado hasta el momento saco las siguientes conclusiones, que más adelante ejemplico con textos y sus comentarios pertinentes.

1) Los elementos B y C - la persona jurídica y el texto de la ley- están en los documentos de todas las fechas y de todos los dialectos. No son, por tanto, pertinentes.

2) Hay dos modelos antiguos: Uno se encuentra en territorio jónico-ático y el otro en zona doria. Este segundo modelo está muy bien documentado e-n las islas dorias del este del Egeo a lo largo del siglo V.

3) El modelo dorio es más arcaizante que el jónico-ático.

4) A lo largo del siglo IV hasta casi finales del III se encuentran dos modelos extendidos por todas las zonas griegas. En estos siglos se introducen dos tipos de innovaciones importantes, ellas son las siguientes:

4.1. Se crean muchas variantes en el orden de los elementos, que llevan al hecho de que aparezca un número muy alto de tipologías distintas, cuyo estudio es interesante desde el punto de vista del estilo.

4.2. Se introducen cuatro elementos que son recientes:

- Solo a partir del siglo IV se encuentra el elemento G. Garantes y el elemento 2. Objeto jurídico.

- El elemento E. Fecha, citada en día y mes, y no solamente mediante el nombre de la persona y el cargo que desempeña, se encuentra desde fines del siglo IV.

- El elemento F. La Finalidad está en los textos a partir del siglo III.

5) A partir del siglo II tiende a imponerse una norma, que consiste en unificar el número de elementos y a darles un orden fijo. Este modelo que tiende a imponerse procede del jonio-ático. A partir de ese momento la estructura sintáctica de los decretos de proxenía se hace menos rígida. Desde el punto de vista sintáctico se encuentran muchos casos de neutralización. Y esto tiende a evitarlo el estilo jurídico y el científico en general.

Las cuatro primeras proxenías que voy a analizar son del V.

Las dos primeras presentan el modelo jónico-ático, con dos variantes unas más simplificada y otra más amplia.

El primer texto consta de los siguientes elementos y en este orden

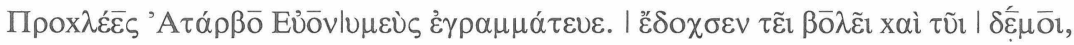

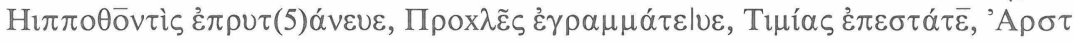




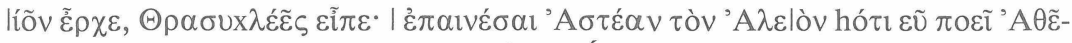

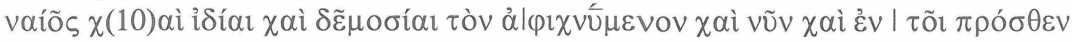

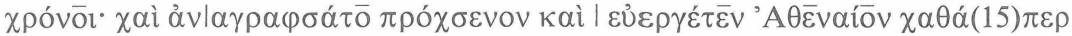

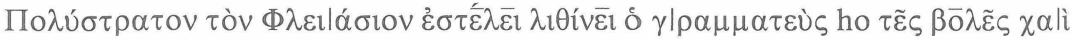

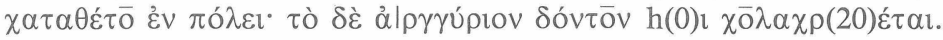

E Fecha expresada mediante el nombre de la persona y su cargo.

$B$ Persona jurídica.

$E$ Recurrencia sin variación: Fecha expresada mediante el nombre de la persona y su cargo.

3 Proponente

$C$ Texto de la ley.

$A$ Causa.

$D$ Ejecución.

Dado que los elementos B y C -Persona juídica y texto de la ley- no son pertinentes, el modelo tiene esta tipología: E.- Fecha, 3.- Proponente, A.- Causa y D.- Ejecución.

El segundo texto es un poco más reciente, del 408. Consta de los siguientes elementos y en este orden

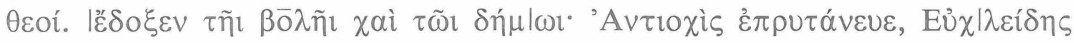

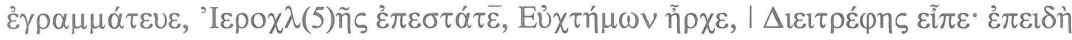

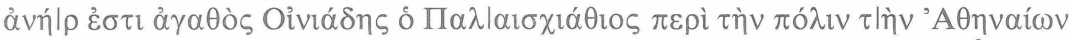

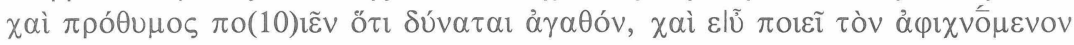

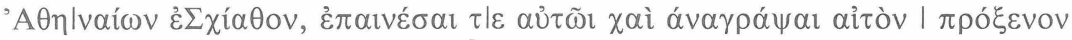

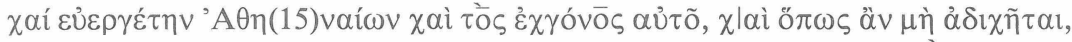

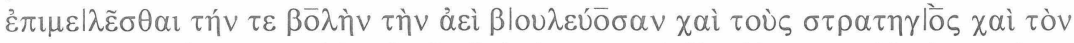

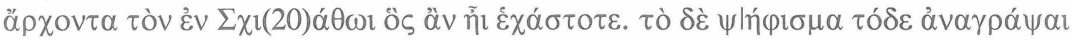

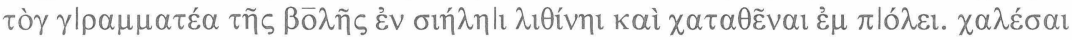

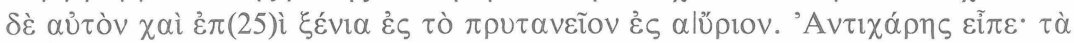

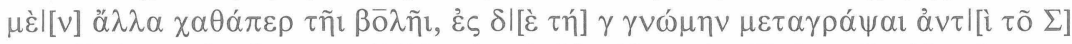

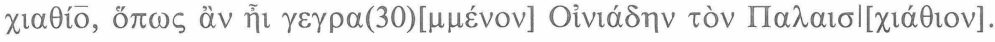

$I$ Invocación.

$B$ Persona jurídica.

E Fecha, citada mediante el nombre de la persona y su cargo.

3 Proponente.

$A$ Causa.

$C$ Texto de la ley.

$D$ Ejecución.

Suprimidos los elementos comunes B y C - Persona jurídica y texto de la ley - tenemos el mismo tipo ampliado por la invocación. El modelo presenta la misma tipología: $I$ Invocación, $E$ Fecha, 3 Proponente, $A$ Causa y $D$ Ejecución.

Los dos textos segundos a que me voy a referir son dorios del este del egeo. Presentan el modelo dorio con dos variantes, una más simplificada y otra más amplia.

El primer texto consta de los siguientes elementos y en este orden 


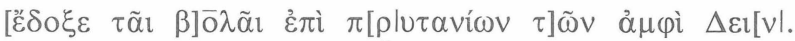
]av $\Pi v \theta \varepsilon \dot{\varepsilon} \omega$

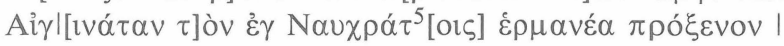

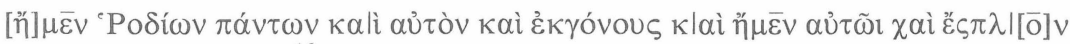

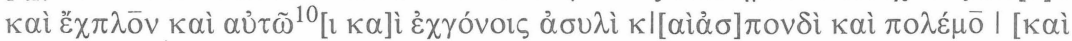
Eip]ńvฤs.

$B$ persona jurídica.

$E$ Fecha mencionada por el nombre de la persona y el cargo que ocupa.

$C$ Texto de la ley.

Como se ve es un modelo que presenta una tipología diferente y muy simplificada, pues tan solo añade a los elementos B y C - persona jurídica y texto de la ley-que son comunes y no pertinentes por tanto, el elemento $E$.

El segundo texto dorio, también del este del Egeo, consta de los siguientes elementos y en este orden

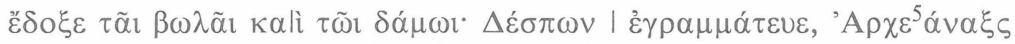

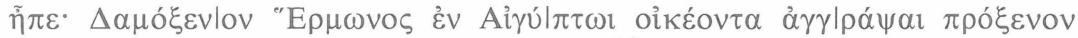

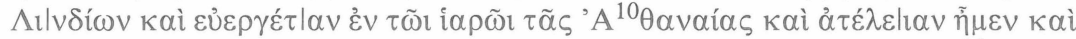

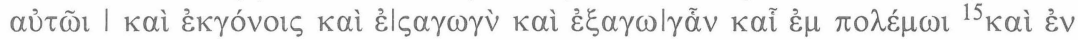

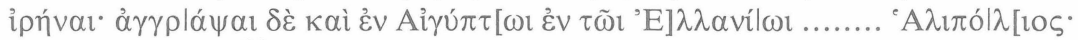

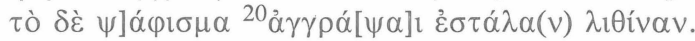

$B$ Persona jurídica.

$E$ Fecha, mencionada con el nombre de la persona y su cargo.

3 Proponente.

$C$ Texto de la ley.

$D$ Ejecución.

Presenta esta tipología: $E$ Fecha, 3 Proponente y $D$ Ejecución.

Como se ve este modelo carece de los elementos $I$ Invocación y $A$ Causa. Responde a un tipo más arcaizante que el modelo jónico-ático de la misma fecha.

La situación normativa a lo largo de los siglos IV y III, cuyas características anunciaba al comienzo, la reflejan bien los textos que voy a analizar a continuación.

$\mathrm{El}$ modelo dorio es frecuente encontrarlo en Beocia y está, entre otros muchos lugares, en Corcira, Argos etc. El texto que paso a comentar es de Tanagra. Consta de los siguientes elementos y en este orden

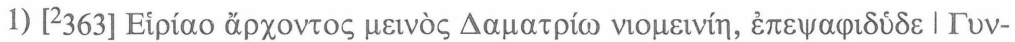

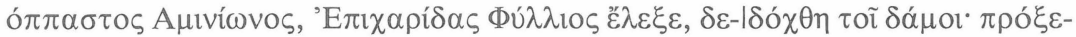

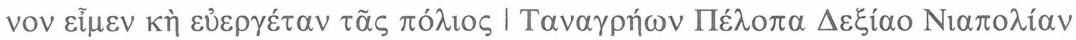

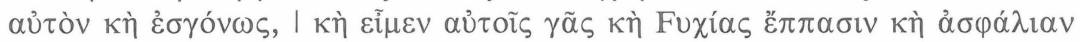

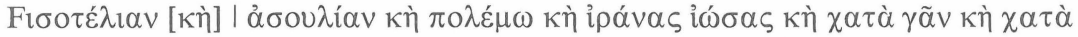

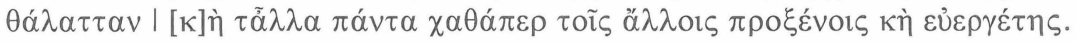

E Fecha, mencionada con el nombre de la persona y su cargo y además el día y el mes.

3 Proponente.

$B$ Persona jurídica.

$C$ Texto de la ley. 
Como se ve han introducido variantes: Alteración en el orden de los elementos y supresión del elemento $D$ Ejecución. Pero la tipología presenta solamente los elementos $E$ Fecha y 3 Proponente. Faltan sistemáticamente los elementos $I$ Invocación y $A$ Causa.

El modelo jónico-ático está extendido por una zona geográfica muy amplia: está en Megara, en Creta, Delfos, en el resto de la zona doria del este del Egeo aparte de Creta, en Beocia, por ejemplo. El texto que sigue es de Megara. Consta de los siguientes elementos y según el siguiente orden

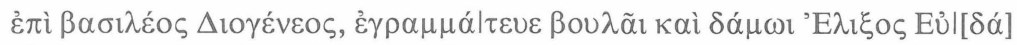

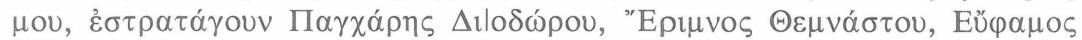

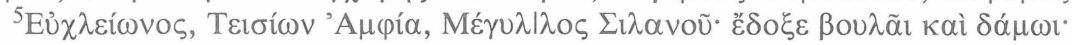

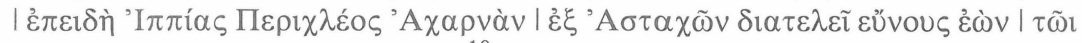

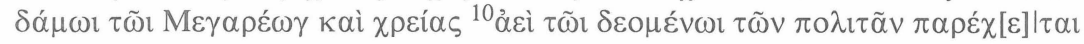

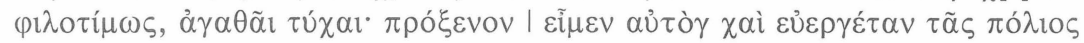

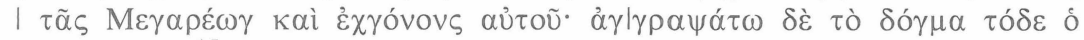

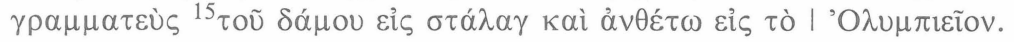

$E$ Fecha, expresada con el nombre de la persona y su cargo.

$B$ Persona jurídica.

$A$ Causa.

I Invocación.

$C$ Texto de la ley.

$D$ Ejecución.

La variante principal que se ha introducido es la variación en el orden de los elementos. A veces se suprimen el elemento 3 Proponente, como aquí, pero esto no sucede siempre, ni es lo más frecuente tampoco. Pero presenta la tipología: $I$ Invocación, $E$ Fecha, $A$ Causa y $D$ Ejecución. O sea, se dan sistemáticamente los elementos $I$ Invocación y $A$ Causa, que faltan en el modelo dorio.

El último texto, que cito a continuación, presenta la tipología que es sistemática en todas las zonas a partir del siglo II. Esta tipología deriva claramente del modelo jónico-ático. Se tiende, por otra parte, a regularizar el número de los elementos y a darles un orden fijo. Como contrapartida la sintaxis se hace menos rígida y se encuentran frecuentes recurrencias, con o sin variación.

El texto consta de los siguientes elementos y en este orden

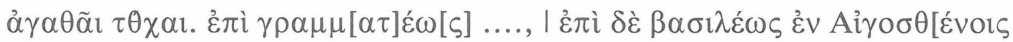

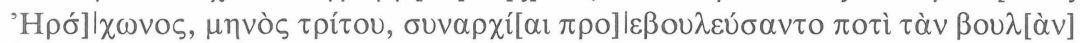

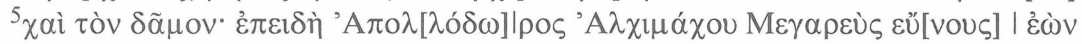

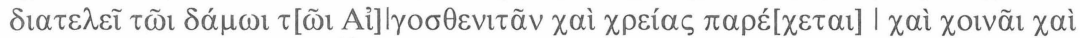

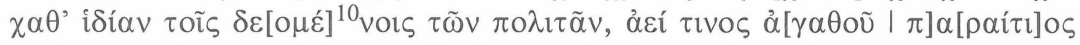

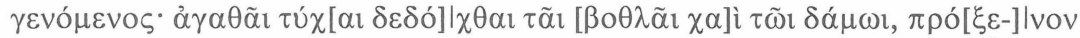

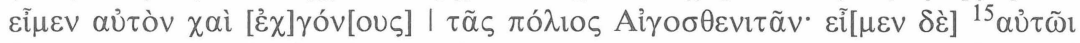

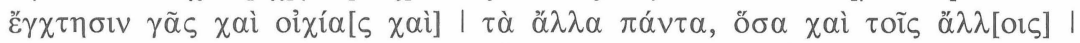

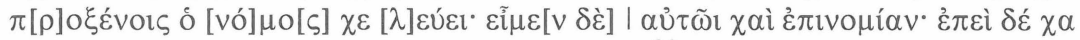

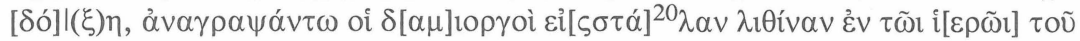

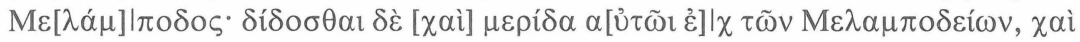

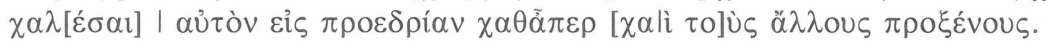


I Invocación.

$E$ Fecha, con el nombre de la persona y el cargo que desempeña, y además el mes.

3 Proponente.

$A$ Causa.

$B$ Persona jurídica, que se introduce con la repetición de la fórmula de la invocación que trasbasada a otro plano de la comunicación, el declarativo, se neutraliza, desempeñando otra funcionalidad.

$C$ Texto de la ley.

$A i$ Causa. Se trata de una recurrencia con variación.

$D$ Ejecución.

$\mathrm{Ci}$ Texto de la ley. Se trata de una recurrencia del elemento $C$ Causa que ahora constituye un expansión.

La tipología que presenta el texto, marginando los elementos que no son pertinentes - B y C- es: $I$ Invocación, $E$ Fecha, 3 Proponente, $A$ Causa y $D$ Ejecución.

Esta tipología, sistemática en el siglo II, consiste en una generalización del modelo antiguo, que ya estaba en zona jonio-ático desde el sigloV a. J.C. 